\title{
Evaluation of Crocins in Cheeses Made with Saffron by UHPLC
}

\author{
Mena Ritota,* Maria Mattera, Maria Gabriella Di Costanzo and Pamela Manzi \\ Consiglio per la ricerca in agricoltura e l'analisi dell'economia agraria, \\ Centro di ricerca Alimenti e Nutrizione (CREA-AN), Via Ardeatina 546, 00178 Rome, Italy
}

\begin{abstract}
Saffron is a widespread consumed spice with potential health promoting compounds. In dairy technologies it is often used to enhance color and flavor of cheeses, so it would be recommended to know the content of saffron functional compounds in cheese made with this spice, as they could still have potential bioactivity. For this purpose, an UHPLC (ultra high performance liquid chromatography) method was developed, with the aim of reducing analysis time and solvent consumption. A methanol:water solution $(80: 20 \mathrm{v} / \mathrm{v})$ was used to extract saffron compounds from cheese with stirring for $1 \mathrm{~h}$ in the dark at room temperature, then the samples were centrifuged at $3500 \mathrm{rpm}$, for $5 \mathrm{~min}$ at $4{ }^{\circ} \mathrm{C}$ and the residues were extracted twice. A linear gradient elution of acetonitrile in water allowed to simultaneously determine picrocrocin and crocins in saffron in a short time (16 min), and allowed a quantitative determination of crocins in commercial cheeses.
\end{abstract}

Keywords: cheese, saffron, crocins, UHPLC

\section{Introduction}

Saffron is the red dried stigmas of Crocus sativus L. flowers, now successfully cultivated in European countries (Greece, Spain, Italy, France and Switzerland) and in Morocco, Egypt, Israel, Azerbaijan, Pakistan, India, New Zealand, Australia and China.

Recently, a review by Akowuah and Htar ${ }^{1}$ reported many therapeutic properties of saffron chemical constituents, such as anticancer, cardioprotective, antidepressant, anxiolytic and anticonvulsant activities, improving learning and memory skills and management of metabolic syndrome diseases. The bioactive compounds mainly responsible of the therapeutic properties of saffron are crocins, crocetin, picrocrocin and safranal. Crocins, responsible for color and coloring properties, are a group of high water soluble cis and trans carotenoids that are sugar esters of crocetin ( $8,8^{\prime}$ '-diapocarotenedioic acid), with different sugar moieties, such as glucose, gentiobiose and neapolitanose. Trans-crocetin di( $\beta$-D-gentiobiosyl) ester is the most abundant crocin in saffron ${ }^{2}$ with a high solubility being attributed to the sugar moieties. The bitter taste of saffron is derived primarily from picrocrocin (4-( $\beta$-D-glucopyranosyloxy)-2,6,6-trimethyl1-cyclohexene-1-carboxaldehyde), a monoterpene glycoside produced from zeaxanthin degradation. Crocins

*e-mail: mena.ritota@crea.gov.it represent from 0.5 to $32.4 \%$ of saffron's dry matter, ${ }^{3}$ while picrocrocin ranges from 0.8 to $26.6 \%$ on a dry matter. ${ }^{3-5}$

Food items containing saffron are generally expected to provide an added value for consumers, thanks to its potential health promoting compounds. For this reason, saffron has been widely studied and many analytical methods for its compounds determination are available: in particular, the standard method, ${ }^{6}$ which specifies the test methods for dried saffron (filaments and powder) obtained from the Crocus sativus L. flower, is based on the use of UV-Vis spectrophotometry to determine the quality of saffron in international commercial agreements, but many other analytical methods were developed, such as: thin layer chromatography, ${ }^{4}$ high performance liquid chromatography with diode array detector, ${ }^{7}$ high performance liquid chromatography coupled with mass spectrometry, ${ }^{8,9}$ near infrared spectroscopy, ${ }^{10}$ capillary electrophoresis, ${ }^{11} \mathrm{UV}$-Vis Fourier transform (FT)-Raman with proton nuclear magnetic resonance spectroscopies. ${ }^{12}$

In dairy technologies saffron is often used in order to enhance color and flavor. Unfortunately, cheeses made with saffron represent only a niche production and often still unknown, for this reason it would be helpful to know the content of saffron functional compounds in cheese, as they could be transfer from saffron to cheese and could still have potential promoting health substances.

Only few works are available in literature about saffron molecules into dairy products, but most of them are 
based on color ${ }^{13,14}$ and volatile compounds ${ }^{15,16}$ of saffron. Therefore, an analytical method focused on cheese made with this spice could be helpful, in order to investigate major functional saffron compounds in this food item. High performance liquid chromatography (HPLC) is the most popular analytical technique for this purpose and, coupled with photodiode array (PDA) detection, it is considered the most efficient method, offering the advantage of isolating crocins $(440 \mathrm{~nm})$, picrocrocin $(250 \mathrm{~nm})$ and safranal (330 nm). Moreover, it can provide the trans/cis-crocins ratio, which is important for saffron therapeutic properties, since they are very often attributed to a specific isomeric form, such as trans-crocins. ${ }^{17,18}$ However, these chromatographic methods showed the disadvantage of general very long time chromatographic runs (about 50-60 min).

The aim of this work was to provide an analytical method able to determine picrocrocin and crocins in cheese made with saffron. Starting from a revisiting extraction of crocins and picrocrocin in saffron powder, extraction procedure was optimized in cheese. An UHPLC (ultra high performance liquid chromatography) method was provided in order to determine these analytes in cheese made with saffron in a very short time and with a minimal solvent consumption.

\section{Experimental}

\section{Samples and chemicals}

Three different Italian cheeses, characterized by the addition of saffron during cheese making, were studied. Three samples of the same cheese batch were analyzed, each one in triplicate.

Cheeses were produced according to local and traditional manufacturing and directly obtained from farms. The first was a soft cheese (A), obtained from ewe milk, produced in the South of Italy; the other two samples were extra hard cheeses (B and C), collected from the same farm in the North of Italy, obtained from cow milk and with different cheese making. All cheeses were sampled and grated according to the ISO|IDF procedures. ${ }^{19}$

Dairy farm, which produced $\mathrm{B}$ and $\mathrm{C}$ cheeses, also provided the saffron $(\mathrm{S} 1)$ used in cheese making. It was saffron powder, $100 \%$ pure, harvested in 2014 in the region of La Mancha, Spain and classified as Category I (quality characteristics determined according to the ISO). ${ }^{6}$ This spice was used for a preliminary optimization of the extraction procedure. Moreover, a commercial dried flower stigmas of saffron (S2), purchased from Sigma (SigmaAldrich Co., St. Louis, MO, USA), was evaluated.

Crocetin di( $\beta$-D-gentiobiosyl) ester (trans-4-GG) and acetonitrile (Chromasolv® Plus, for HPLC, $\geq 99.9 \%$ ) were obtained from Sigma. Methanol (special grade, RS - for UHPLC-MS) was from Carlo Erba (Carlo Erba Reagents S.A.S., Val de Reuil, France); water was purified through a Milli-Q system (ion exchange system to $>18 \mathrm{~m} \Omega \mathrm{cm}$ resistivity, Millipore, Billerica, MA, USA).

All solvents were filtered through $0.2 \mu \mathrm{m}$ membrane filters (Phenomenex Inc., Torrance, CA, USA).

\section{Extraction procedure on saffron powder}

The extraction of functional components from saffron was optimized as a function of extraction time and solvent extraction system. Three different extraction times (by stirring) were studied (1, 3 and $5 \mathrm{~h}$ ); after selecting the optimum extraction time, some solvents (water, ethanol, methanol, acetone) and their mixture with water (50:50, v/v and 80:20, v/v) were evaluated for $1 \mathrm{~h}$ stirring times.

The optimized extraction procedure was: stirring $10 \mathrm{mg}$ of saffron powder with $10 \mathrm{~mL}$ of a mixture of methanol:water $(80: 20, \mathrm{v} / \mathrm{v})$, in the dark and at room temperature for $1 \mathrm{~h}$.

\section{Extraction procedure on cheeses made with saffron}

This optimized extraction was also applied to cheese, taking into account the matrix influence on extraction; therefore more extractions, in function of extraction times by stirring, were evaluated in cheeses: $(i)$ three extractions, $1 \mathrm{~h}$ each; (ii) three extractions of 60, 20 and $20 \mathrm{~min}$, respectively; (iii) three extractions of 40, 20 and $20 \mathrm{~min}$, respectively. In every case, after the extractions, the samples were centrifuged at $3500 \mathrm{rpm}$, for $5 \mathrm{~min}$ at $4{ }^{\circ} \mathrm{C}$; the supernatants were then collected and the residual samples were extracted twice, according to the extraction time established.

The optimized procedure for cheese with saffron was: $2.5 \mathrm{~g}$ of grated cheese extracted with $10 \mathrm{~mL}$ of methanol:water $(80: 20, \mathrm{v} / \mathrm{v})$ by stirring in the dark and at room temperature for $1 \mathrm{~h}$; then sample was centrifuged at $3500 \mathrm{rpm}$, for $5 \mathrm{~min}$ at $4{ }^{\circ} \mathrm{C}$ and the supernatant fraction was collected. Extraction was repeated twice more in the same way and the collected supernatant fractions were filled to the $30 \mathrm{~mL}$ mark with methanol:water $(80: 20, \mathrm{v} / \mathrm{v})$. Before UHPLC-PDA analysis, the extract was filtered $(0.2 \mu \mathrm{m})$.

\section{UHPLC equipment and conditions}

Chromatographic separation was performed on a Nexera UHPLC system (Shimadzu Corporation, Kyoto, Japan) 
equipped with two LC-30AD pumps, UV-Vis SPD-M20A photodiode array detector and SIL 30-AC autosampler, using a Synergi Fusion-RP $(100 \times 2.0 \mathrm{~mm}, 2.5 \mu \mathrm{m}$, Phenomenex Inc.) column with a MercuryMS ${ }^{\mathrm{TM}}$ LC/MS cartridge $(20 \times 2.0 \mathrm{~mm}$, Phenomenex Inc.). LabSolution Version 5.42 SP5 (Shimadzu Corporation) software was used for equipment control, data acquisition and chromatographic data processing.

The selected elution system was a gradient of acetonitrile in water from 15 to $55 \%$ in $10.50 \mathrm{~min}, 55 \%$ acetonitrile for $1 \mathrm{~min}$, from 55 to $15 \%$ acetonitrile in $0.5 \mathrm{~min}$ and a re-equilibration time of $4 \mathrm{~min}$; flow rate $0.4 \mathrm{~mL} \mathrm{~min}^{-1}$; PDA detector (set from 190 to $800 \mathrm{~nm}$ ) with simultaneously chromatograms recording at $\lambda=250 \mathrm{~nm}$ (picrocrocin) and $\lambda=440 \mathrm{~nm}$ (crocins).

Total crocins content, expressed as $\mathrm{mg} 100 \mathrm{~g} \mathrm{~g}^{-1}$ of cheese, was calculated by means of external calibration curve with trans-4-GG standard (68\% purity), supposing the same response factor for all trans-crocins. Moreover, due to the limited availability of the standards and considering their low presence in cheese, the same response factor was used for cis-crocins. Hence, total crocins content in cheese represents the sum of all cis and trans-crocins, expressed as trans-4-GG content.

\section{Method validation}

In order to verify the linearity range, a calibration curve was performed by injecting different concentrations of trans-4-GG standard solutions; each of the five calibration levels $\left(0.72,1.44,7.18,14.36\right.$ and $\left.28.72 \mu \mathrm{g} \mathrm{mL}^{-1}\right)$ was analyzed in triplicate.

Limit of detection (LOD) and limit of quantification (LOQ), performed on direct injection of crocins standards, were calculated as follows:

$\mathrm{LOD}=\mathrm{Xb}+3 \mathrm{SDb}$

$\mathrm{LOQ}=\mathrm{Xb}+10 \mathrm{SDb}$

where $\mathrm{Xb}$ was the blank mean value $(\mathrm{n}=10)$ and $\mathrm{SDb}$ the blank standard deviation. ${ }^{20}$

Precision of the method was determined through measurements of repeatability and reproducibility on direct injection of crocins standard and was expressed in terms of relative standard deviation (RSD): the intra-day variability (repeatability) was measured on three replicates, while the inter-day variability (reproducibility) was evaluated on three separate days.

Accuracy of the method was evaluated by spiking three different samples of grated cheeses with a known amount of trans-4-GG prior to extraction. The recovery was a measurement of accuracy (\%) and it was calculated as follows:

$$
\text { Recovery }(\%)=\frac{\text { mean concentration measured }}{\left(\begin{array}{c}
\text { mean concentration of non spiked } \\
\text { sample }+ \text { spiked concentration })
\end{array}\right.} \times 100
$$

Recovery was a mean of two different replicates on three different cheeses.

\section{Statistics}

Statistical analysis was performed using the PAST 2.17 software. ${ }^{21}$ The null hypotheses of normality and homogeneity of variance were tested using Shapiro-Wilk's test and Levene's test, respectively. Significant differences of the mean values were evaluated by one-way analysis of variance (ANOVA), followed by a Tukey's post hoc with Bonferroni correction. Friedman and Kruskal-Wallis tests were instead applied to test significant differences among data with non normal distribution and with non homogeneity of variance, respectively.

\section{Results and Discussion}

\section{Optimization of extraction procedure on saffron powder}

The extraction of functional components from saffron was optimized as a function of extraction time (by stirring) and solvent through the "one factor at a time" approach. A schematic representation is depicted in Figure 1.

\section{Extraction time}

Extraction by stirring and room temperature were chosen according to the standard method for the saffron quality determination ${ }^{6}$ and Lozano et al.,$^{22}$ where $25^{\circ} \mathrm{C}$ was the optimal temperature for saffron compounds extraction.

Different extraction times, 1,3 and $5 \mathrm{~h}$, with a mixture of methanol:water (80:20) were tested to evaluate the extraction efficiency. Three repetitions were carried out for each condition. In order to avoid degradation, long extraction times, such as $24 \mathrm{~h},{ }^{3,23}$ were not considered. Optimization of the extraction time was carried out by using a saffron weight:solvent volume ratio equal to $1: 1$. After 1, 3 and $5 \mathrm{~h}$, aliquots of each extract were filtered and analyzed by UHPLC with PDA detector for the picrocrocin and crocins content. The areas of the most intense peaks, later identified as trans-4-GG, trans-3-gG and picrocrocin, and the sum of the total areas of the saffron crocins, identified according to the appearance of their UV-Vis spectra, were monitored to evaluate the efficiency of the extraction time. 
- SAFFRON -

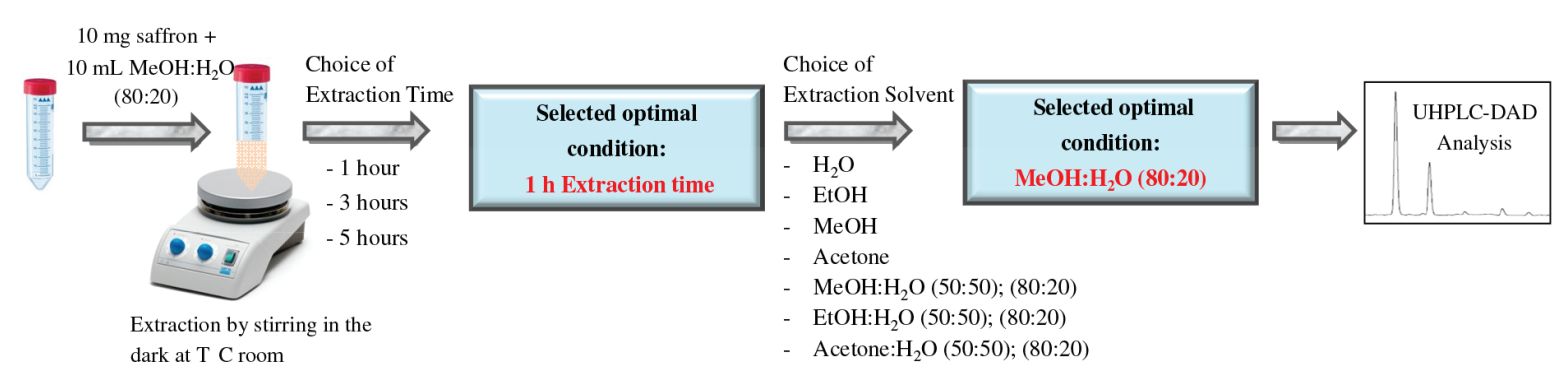

- CHEESE -

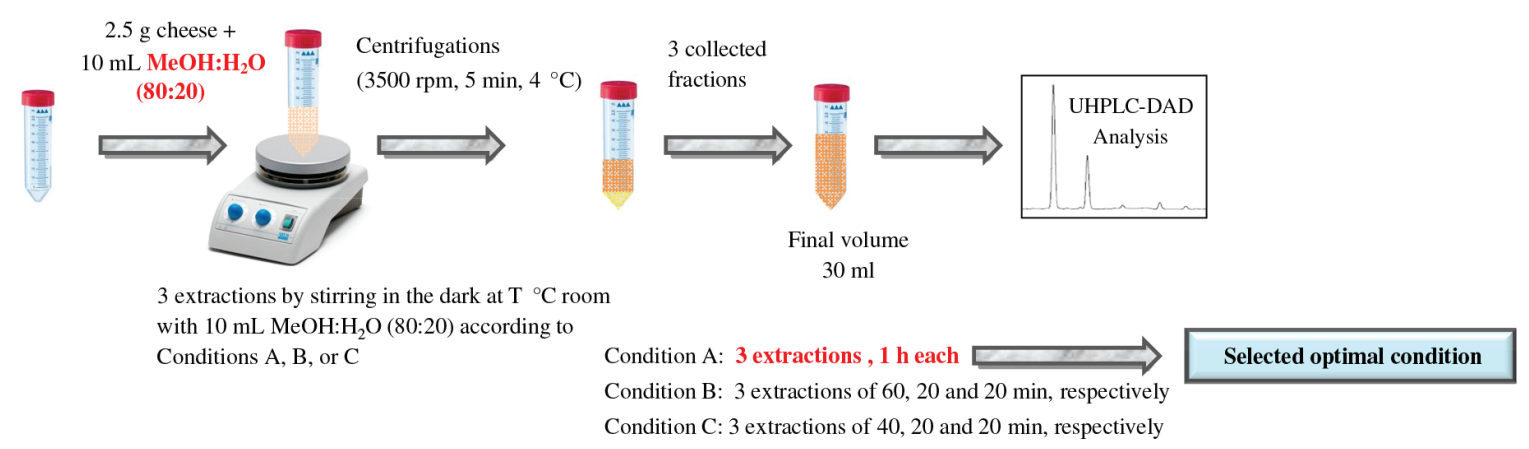

Figure 1. Schematic representation of saffron and cheese optimization extractions.

Figure 2A shows a decreasing trend of the area from 1 to $3 \mathrm{~h}$ extraction times for all the peaks evaluated: $10 \%$ for trans-4-GG and trans-3-gG, 5\% for picrocrocin and $9 \%$ for the total crocins. After $5 \mathrm{~h}$ extraction time, a further percentage point decrease was observed for trans-4-GG and the total crocins. Statistical analysis revealed that there was a significant difference $(p<0.05)$ on the mean values only in the case of picrocrocin: $1 \mathrm{~h}$ extraction time significantly differed $(p<0.01)$ from 3 and 5 h extraction time (Figure 2A).

These results confirmed the evidence of Kyriakoudi et al. ${ }^{24}$ according to which, through a statistical approach (response surface methodology, RSM), extraction time had the strongest influence on the picrocrocin content and no significant effect on crocins, compared to the type of solvent and type of extraction. Sarfarazi et al. ${ }^{25}$ instead, applied RSM to optimize ethanol concentration, extraction time and temperature, showing that extraction maceration time was significant on picrocrocin and crocins response only through interactive effects; however, the same authors admitted the low predictive capability of their model and that their RSM algorithm overestimated all responses.

According to our results, a $1 \mathrm{~h}$ stirring time with a mixture of methanol:water (80:20) was selected as the condition with the highest efficiency to extract crocins and picrocrocin from saffron powder.

\section{Solvent extraction system}

Different solvents (water, ethanol, methanol and acetone) and their mixture with water (50:50, v/v and $80: 20, \mathrm{v} / \mathrm{v}$ ) were studied for $1 \mathrm{~h}$ extraction time by stirring. Three repetitions were carried out for each condition. This choice was based on previous works: water was undoubtedly the most widely used solvent to extract compounds from saffron, ${ }^{3,6,26}$ but other solvents, such as methanol, ${ }^{27}$ ethanol ${ }^{4}$ or their mixture, were used. ${ }^{24,28,29}$

In Figure 2B the areas of the total crocins of saffron extracts in function of the extraction solvent are reported. According to these results mixture of the different solvents with water obtained better results than the solvents alone, confirming in part the data of Orfanou and Tsimidou, ${ }^{30}$ according to which water:alcohol mixtures had better extraction efficiency than water or alcohol alone. Acetone was the worst extraction solvent, confirming that it is not suitable for the extraction of the water soluble carotenoids, such as crocins. The highest areas were obtained with a mixture of methanol:water $(80: 20, \mathrm{v} / \mathrm{v})$, followed by mixtures of acetone:water $(50: 50, \mathrm{v} / \mathrm{v})$ and ethanol:water $(50: 50, \mathrm{v} / \mathrm{v})$. This was not in agreement with Kyriakoudi et al. ${ }^{24}$ results, who reported a mixture of methanol:water (50:50) as optimal extraction solvent for saffron crocins. However, the same authors tested the extraction mixture with another extraction methodology, 
A

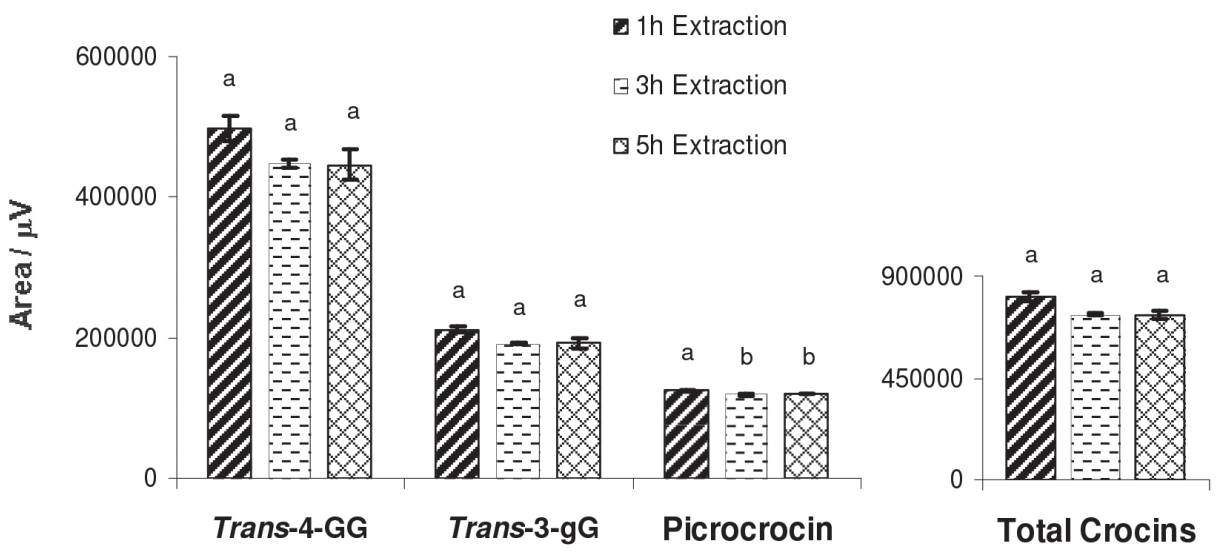

B

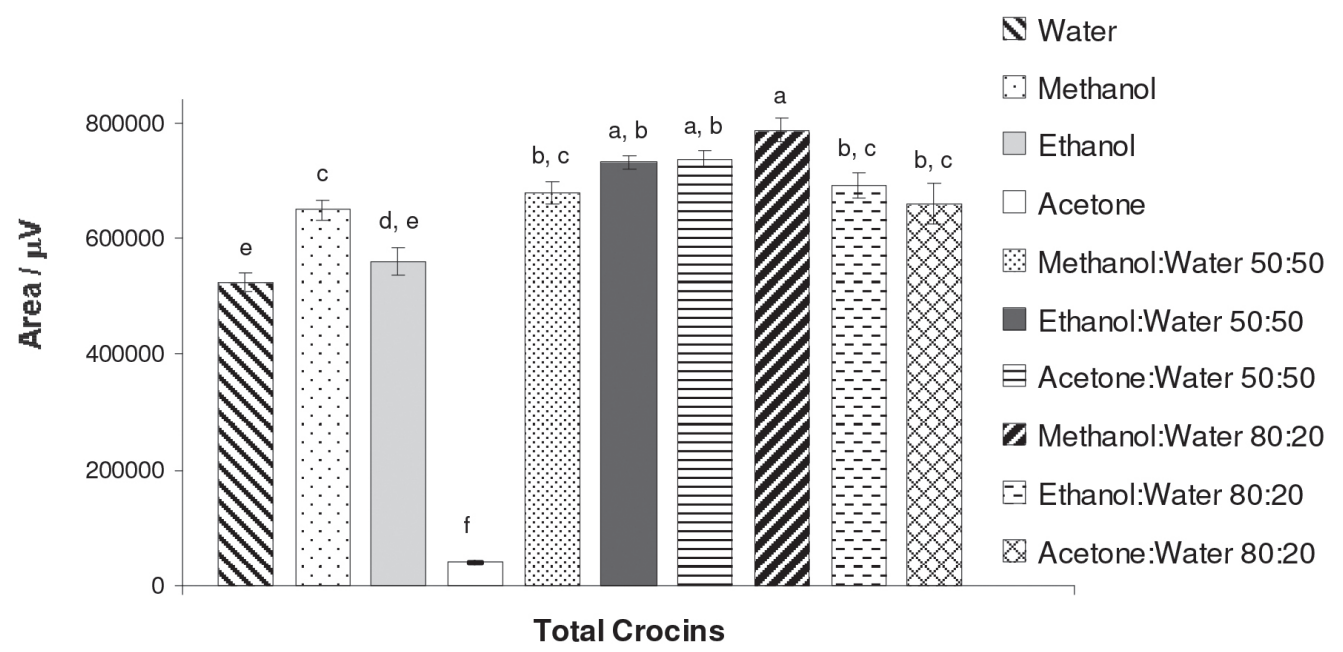

Figure 2. (A) Saffron (S1) extracts in methanol:water (50:50, v/v) as a function of stirring time (areas mean of three different injections); (B) $1 \mathrm{~h}$ stirring time of saffron (S1) extracts as a function of extraction solvent (areas mean of three different injections). For each compound, significant differences $(p<0.05)$ were identified with different letters.

i.e. sonication, which encourages membrane rupture and cell lysis, ${ }^{31}$ enhancing methanol penetrating ability into the sample matrix.

One-way ANOVA on the mean values of the total crocins areas revealed a high significant difference $(p<0.01)$ among the several extraction solvents (Figure 2B). A Bonferroni correction on Tukey's test showed that the three solvents with the highest extraction efficiency, methanol:water $(80: 20, \mathrm{v} / \mathrm{v})$, acetone:water $(50: 50, \mathrm{v} / \mathrm{v})$ and ethanol:water $(50: 50, \mathrm{v} / \mathrm{v})$, did not significantly differ $(p<0.05)$ among each other. However, the mixture methanol:water $(80: 20, \mathrm{v} / \mathrm{v})$ was significantly different $(p<0.01)$ from the rest of the solvents tested (Figure 2B), therefore, it was preferred. The same results obtained for total crocins content were observed for the areas of the each peak (trans-4-GG, trans-3-gG and picrocrocin) (data not shown). Regarding the picrocrocin content, a Bonferroni correction on Tukey's test revealed that the most intense area, obtained with methanol:water $(80: 20, \mathrm{v} / \mathrm{v})$, significantly differed $(p<0.01)$ from all the other solvents. However, Kyriakoudi et al. ${ }^{24}$ reported methanol $0.44 \%$ as optimal extraction solvent for saffron picrocrocin applying RSM, but the same authors used a different extraction and the response used to optimize RSM algorithm, based on E1\%257 (picrocrocin response), did not give an accurate measurement of picrocrocin content, due to the trans and cis-crocins interference at $250 \mathrm{~nm} \cdot{ }^{26}$

According to the obtained results, the procedure for saffron crocins and picrocrocin extraction was: stirring $10 \mathrm{mg}$ of saffron powder with $10 \mathrm{~mL}$ of a mixture of 
methanol:water $(80: 20, \mathrm{v} / \mathrm{v})$, in the dark and at room temperature for $1 \mathrm{~h}$.

\section{Extraction procedure on cheeses made with saffron}

The same conditions optimized for saffron were used to extract picrocrocin and crocins from cheese. However, due to the complexity of this matrix, the possibility of using a great number of extractions was considered. In this extraction procedure on cheeses made with saffron, acid or alkaline hydrolysis was not considered, because it could have resulted in a detachment of the sugar moieties of the crocins esters. A schematic representation of extraction procedure in cheese is depicted in Figure 1.

To evaluate the best extraction conditions in cheese extracts, the sum of the total areas of crocins was monitored, because picrocrocin peak was not identified: this molecule is in fact subject to hydrolysis giving safranal and, if saffron is added to milk or curd during cheese making, it could go through degradation.

According to our results (data not shown), three extractions, 1 hour each, were selected. In the 60-20-20 min extraction, in fact, there was a loss of $7 \%$ in the recovery compared to three extractions of $1 \mathrm{~h}$ each, while for the 40-20-20 min extraction there was a loss of $11 \%$. The choice of three extractions, 1 hour each, was also verified by adding a $0.14 \mathrm{mg} \mathrm{mL}^{-1}$ standard crocin solution to cheese prior to extraction, resulting in a $106 \%$ recovery.

Since cheese is a complex matrix, the protein and fat-free samples, by treatment with Carrez reagents, ${ }^{32}$ were also evaluated for the crocin content (data not shown): the results were in agreement with those obtained in the non treated samples, so the authors preferred to avoid using other solvents for sample pretreatment (not eco-friendly).

Optimization of chromatographic conditions and crocins identification

Figure 3 shows UHPLC separation of saffron crocins obtained with different elution systems on a Synergi Fusion-RP column and detected at $440 \mathrm{~nm}$ (max $\lambda$ of crocins). Three different mobile phases: methanol/water, (methanol:acetonitrile)/water and acetonitrile/water, were evaluated in the several gradient elution programs, with a

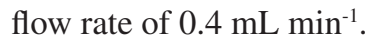

Chromatogram A (Figure 3) employed a methanol/ water solvent system at $25^{\circ} \mathrm{C}$, using the following gradient program: from 25 to $70 \%$ of methanol in $10 \mathrm{~min} ; 70 \%$ methanol for $1 \mathrm{~min}$; from 70 to $25 \%$ of methanol in $0.5 \mathrm{~min}$ and 5 min of re-equilibration time. The main peaks assigned to crocins were well resolved, but the k' (capacity factor) values were higher, ${ }^{33}$ with 8.1 and 13.8 for the shortest and longest retained compounds, respectively. The widths at $50 \%$ peak height for the most intense peaks were in the order of magnitude of few seconds, as expected for UHPLC peaks, that is $0.075 \mathrm{~min}$.

In order to decrease the $\mathrm{k}$ ' values and not to increase the flow rate, methanol was substituted with methanol:acetonitrile $(50: 50, \mathrm{v} / \mathrm{v})$, using the above mentioned elution gradient, (Figure 3, chromatogram B), as already exploited by Caballero-Ortega et al.: ${ }^{23} \mathrm{a}$ better resolution was achieved in the first part of the

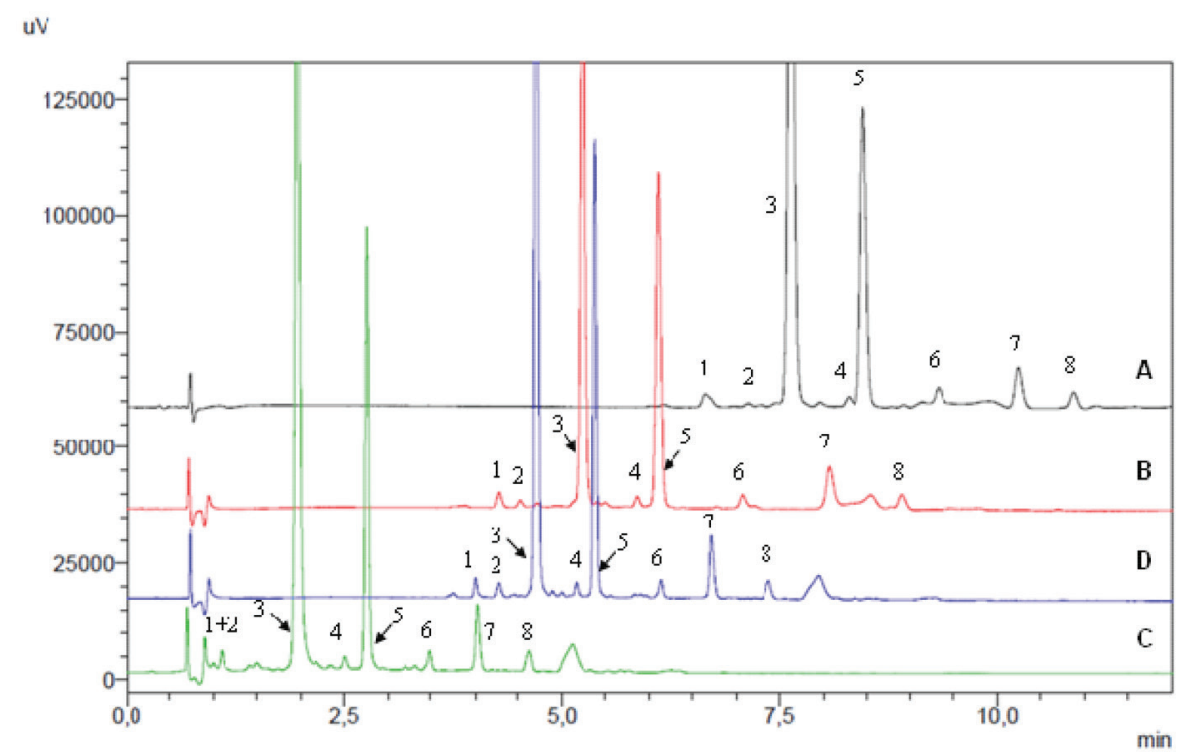

Figure 3. UHPLC chromatograms at $\lambda=440 \mathrm{~nm}$ of a saffron extract (S1) obtained with different mobile phases: (A) methanol/water; (B) (methanol:mcetonitrile) (50:50)/water; (C) and (D) two different gradients of acetonitrile/water. For the elution gradients see Optimization of chromatographic conditions and crocins identification section. trans-Crocins (peaks 1, 2, 4, 6); trans-4-GG (peak 3); trans-3-gG (peak 5); cis-crocins (peaks 7, 8). 
chromatogram, with the appearance of other peaks, early deconvoluted, and a noticeable decrease of the k' values was observed (5.0 and 11.4 for the shortest and longest retained compounds, respectively). Furthermore, the sharpest peaks were obtained with a width at $50 \%$ peak height of 0.058 and $0.061 \mathrm{~min}$, respectively, together with an improvement of the asymmetry values to 1.10 and 1.05 , compared to the previous 1.20 and 1.21 with the methanol/water elution system (Figure 3, chromatogram A).

In chromatogram $C$ (Figure 3 ), using the same elution gradient of chromatograms $\mathrm{A}$ and $\mathrm{B}$ and replacing methanol:acetonitrile $(50: 50, \mathrm{v} / \mathrm{v})$ with acetonitrile, the widths at $50 \%$ peak height for the most intense peaks improved ( 0.057 and $0.043 \mathrm{~min}$, respectively). However, the k' values of the shortest retained compounds were lower (ca. 2), with the retention time of the picrocrocin peak at the recording wavelength of $250 \mathrm{~nm}$ too close to the dead time.

In order to improve the separation efficiency, the elution gradient was optimized (Figure 3, chromatogram D) according to the following program: linear gradient elution of acetonitrile in water from 15 to $55 \%$ in $10.5 \mathrm{~min}$; $55 \%$ acetonitrile for $1 \mathrm{~min}$; from 55 to $15 \%$ acetonitrile in $0.5 \mathrm{~min}$ and $4 \mathrm{~min}$ of re-equilibration time (flow rate $0.4 \mathrm{~mL} \mathrm{~min}^{-1}$ ). In these conditions, the most intense peaks in the chromatogram had an asymmetry value of 1.10 and a width at $50 \%$ peak height of 0.040 and $0.041 \mathrm{~min}$, respectively. Moreover, all detected crocins had a resolution value higher than 1.5 and a $\mathrm{k}$ ' value between 4.5 and 9.1, according to the recommended chromatographic parameters. ${ }^{33}$

An UHPLC chromatogram (Figure 4A) of saffron extract (with methanol:water 80:20 for 1 hour stirring), recorded at 250 and $440 \mathrm{~nm}$, and the UV-Vis spectra of picrocrocin and some crocins (Figure 4B) are showed using the optimized gradient.
Peak identified with asterix at $\lambda=250 \mathrm{~nm}$ was assigned to picrocrocin according to literature ${ }^{3,9}$ and its UV spectrum, which exhibits a characteristic broad absorption band at $250 \mathrm{~nm}$ due to the $\alpha$ - $\beta$-unsaturated cycloaldehyde in the molecule.

The PDA UV-Vis spectra (190-800 nm) of each peak in the chromatogram acquired at $\lambda=440 \mathrm{~nm}$ allowed to identify both trans and cis-crocins. As well known, ${ }^{9}$ the UV-Vis spectra of all trans glycosidic carotenoids show two absorption bands: the first, at 250-260 nm, has the lowest absorptivity and corresponds to the glucosyl ester bonds of crocins, while the second, between 400 and $500 \mathrm{~nm}$, has the highest absorptivity ( $\lambda \max 440 \mathrm{~nm})$ and it is characteristic of all trans carotenoids. The cis isomers, in addition to the two absorption bands of trans isomers, show another band $(\lambda=325 \mathrm{~nm}$ ), attributed to the presence of cis double bonds in the polyene conjugated system of crocins. Therefore, according to the appearance of their UV-Vis spectra, in the saffron chromatogram at $\lambda=440 \mathrm{~nm}$ (Figure 4A), peaks $1,2,3,4,5$ and 6 were attributed to trans-crocins, while peaks 7 and 8 , which represent $5 \%$ of the total crocins content, were assigned to cis-crocins. Furthermore, peak 3 was identified as trans-4-GG, comparing its UV-Vis spectrum and retention time with the standard, while peak 5 was tentatively assigned to trans-3-gG. Many studies, in fact, showed that these crocins are the most abundant in saffron, ${ }^{23,26,28}$ so it is reasonable to think that the second most abundant crocin in the chromatogram at $\lambda=440 \mathrm{~nm}$ of a saffron extract does correspond to trans $-3-\mathrm{gG}$, also according to its UV-Vis spectrum.

According to the chromatographic conditions set above and taking into account the standard purity, a calibration curve for trans-4-GG quantification was used. A linearity range from 0.72 to $28.72 \mu \mathrm{g} \mathrm{mL}^{-1}$ was observed

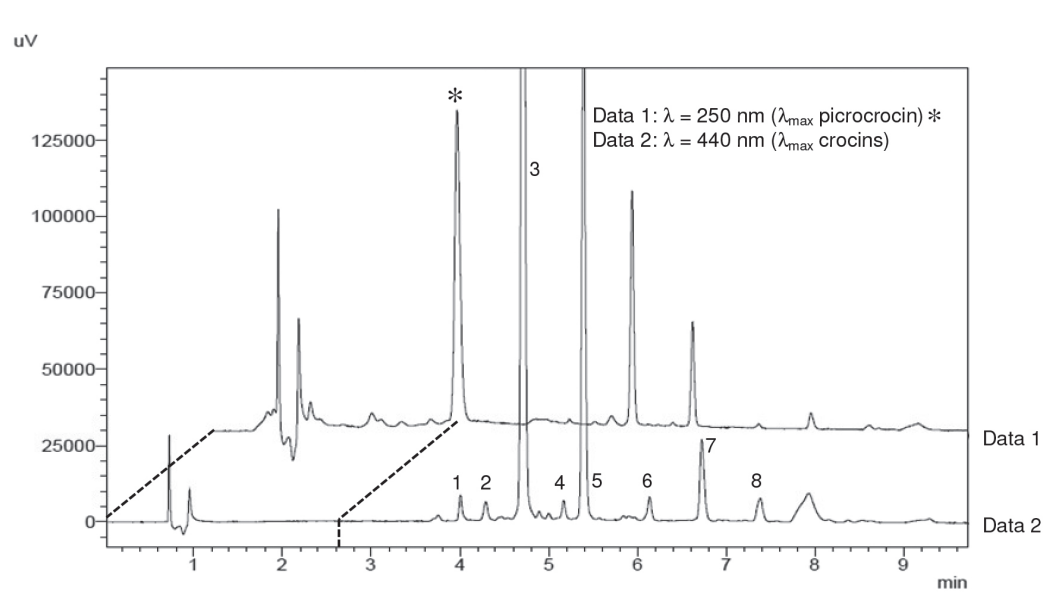

A)

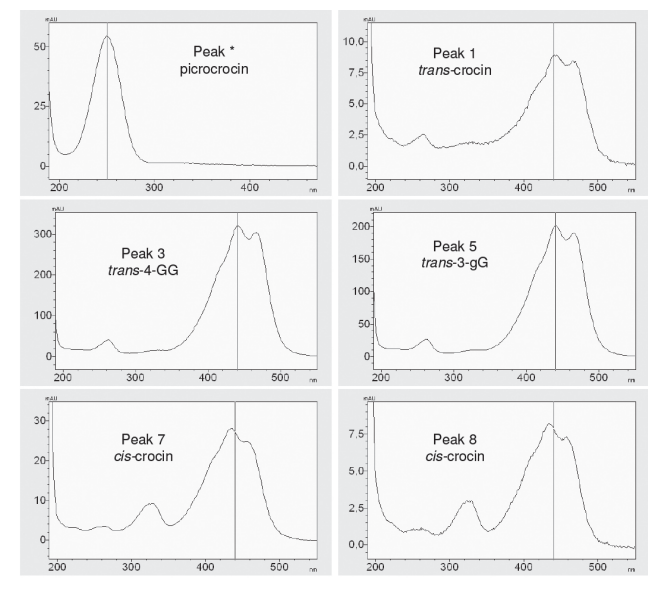

B)

Figure 4. (A) UHPLC chromatograms of saffron (S1) extract detected at 250 and $440 \mathrm{~nm}$ : picrocrocin (peak *); trans-crocins (peaks 1, 2, 4, 6); trans-4-GG (peak 3); trans-3-gG (peak 5); cis-crocins (peaks 7, 8); (B) UV-Vis spectra of picrocrocin and some crocins in saffron (S1) extract. 
for trans-4-GG, obtaining a slope value of 1068.16. Additionally, the $\mathrm{R}^{2}$ (coefficient of determination) value was 0.9999 , with the slope RSD value lower than $1 \%$, which indicated a high accuracy of the method.

LOD and LOQ were $0.07 \mu \mathrm{g} \mathrm{mL}^{-1}$ (or

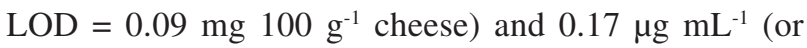

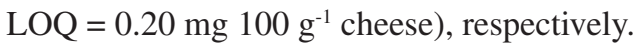

The developed method resulted in good repeatability $\left(\mathrm{n}=3\right.$ at $\left.1.44 \mu \mathrm{g} \mathrm{mL}^{-1}\right)$, with overall intra- and inter-day RSD values of 1.95 and $5.57 \%$, respectively.

A summary of the method performances is reported in Table 1.

\section{Crocins in cheeses made with saffron}

In Figure 5, the UHPLC chromatograms $(\lambda=440 \mathrm{~nm})$ of an extract of saffron (S1), commercial saffron (S2), ewe milk cheese extract (A) and two cow milk cheeses extracts (B and C) are reported. The identification of crocins and picrocrocin in cheese was made by comparing the chromatograms and the UV-Vis spectra of the cheese extracts to those of S1 and S2 (powder saffron and stigmas, respectively), differing in their technological process.
Chromatographic profiles of all cheese samples reflected the chromatographic profile of saffron, differing only in quantitative composition: trans-4-GG and trans-3-gG were the most abundant crocins in cheeses, ranging between $57-64 \%$ of the total crocins content for trans-4-GG and $27-36 \%$ of the total content for trans-3-gG.

The total crocins content (as the sum of all cis and trans-crocins), expressed as trans-4-GG content,

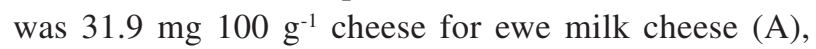
$2.9 \mathrm{mg} 100 \mathrm{~g}^{-1}$ cheese for cow milk cheese (B) and $1.4 \mathrm{mg} 100 \mathrm{~g}^{-1}$ cheese for cow milk cheese (C). The observed differences $(p<0.05)$, verified also in dry matter results, probably depend on the amount of added saffron and/or to different ripening time of the cheeses.

The cis isomers were present only in two cheeses and represented $9 \%$ of the total content for the ewe milk cheese (A) and $10 \%$ of the total content for the cow milk cheese (B). The presence of cis-crocins in cheeses could be due both to their content in the added saffron and to the cis-trans isomerization reaction, promoted by light, ${ }^{34}$ during the cheese making.

As already mentioned, crocins show lowest absorptivity at $250-260 \mathrm{~nm}$ in their UV spectra. Figure 6 reports the same UHPLC chromatograms of Figure 5, but recorded at

Table 1. Summary of the method performances

\begin{tabular}{lcccccc}
\hline & $\begin{array}{c}\text { Linearity range / } \\
(\mu \mathrm{g} \mathrm{mL})\end{array}$ & $\begin{array}{c}\text { LOD / } \\
\left(\mu \mathrm{g} \mathrm{mL}^{-1}\right)\end{array}$ & $\begin{array}{c}\text { LOQ / } \\
\left(\mu \mathrm{g} \mathrm{mL}^{-1}\right)\end{array}$ & $\begin{array}{c}\text { Intra-day } \\
\text { precision }^{\mathrm{a}, \mathrm{b}} / \%\end{array}$ & $\begin{array}{c}\text { Inter-day } \\
\text { precision }^{\mathrm{a}, \mathrm{b}} / \%\end{array}$ & $\begin{array}{c}\text { Recovery }^{\mathrm{b}} / \\
\%\end{array}$ \\
\hline trans-4-GG & $0.72-28.72$ & 0.07 & 0.17 & 1.95 & 5.57 & 106 \\
\hline
\end{tabular}

${ }^{\mathrm{a}} \mathrm{n}=3$; bat $1.44 \mu \mathrm{g} \mathrm{mL}{ }^{-1}$. LOD: limit of detection; LOQ: limit of quantification.

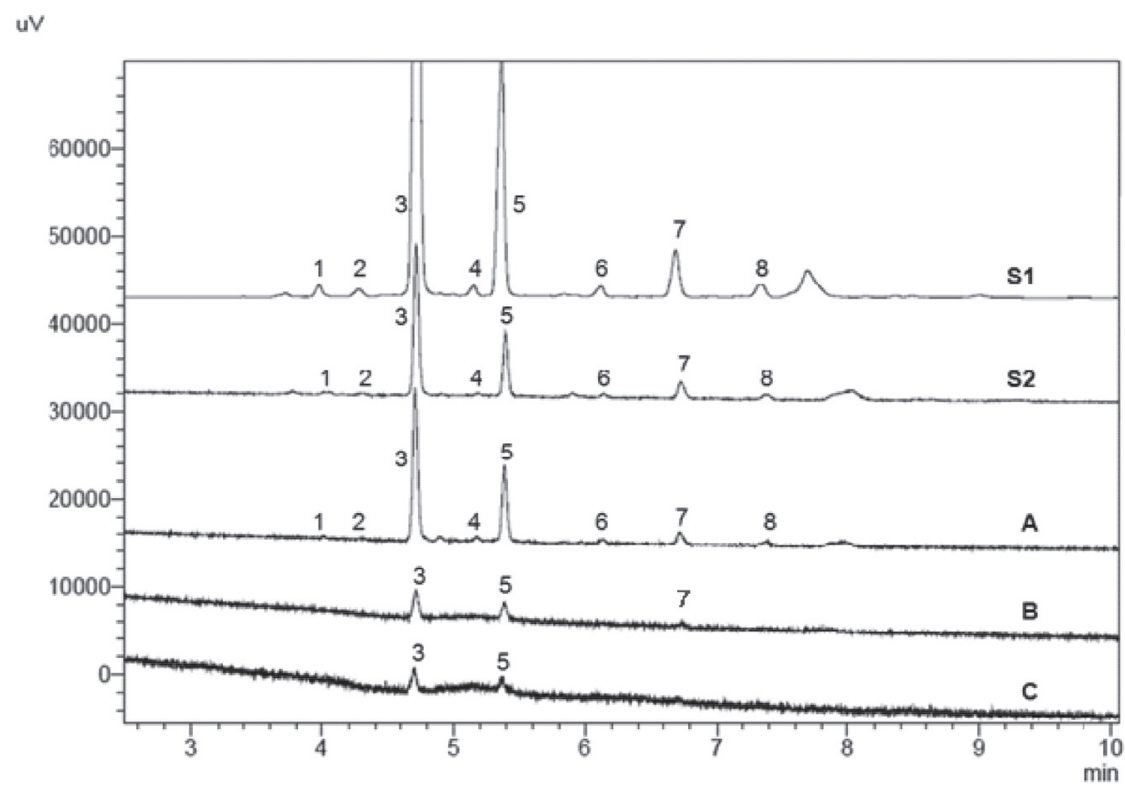

Figure 5. UHPLC chromatograms at $\lambda=440 \mathrm{~nm}$ of extract of: saffron (S1 and S2); ewe milk cheese (A); cow milk cheese (B); cow milk cheese (C). trans-Crocins (peaks 1, 2, 4, 6); trans-4-GG (peak 3); trans-3-gG (peak 5); cis-crocins (peaks 7, 8). 


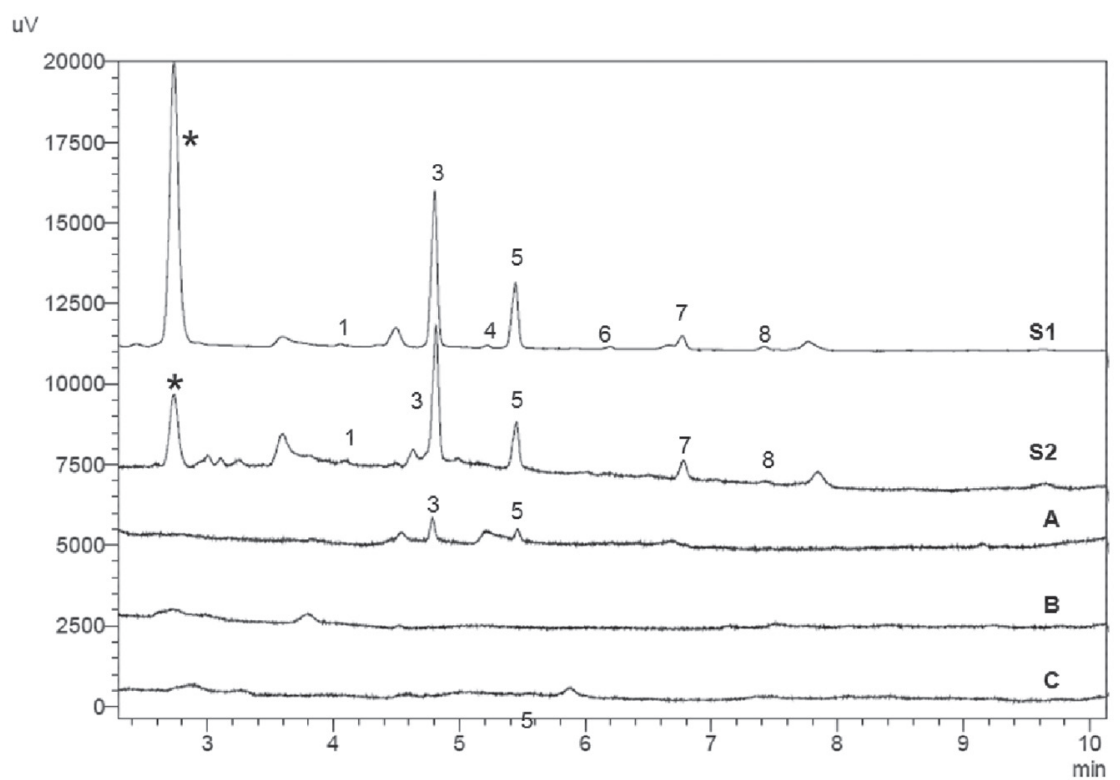

Figure 6. UHPLC chromatograms at $\lambda=250 \mathrm{~nm}$ of extract of: saffron (S1 and S2); ewe milk cheese (A); cow milk cheese (B); cow milk cheese (C). trans-Crocins (peaks 1, 2, 4, 6); trans-4-GG (peak 3); trans-3-gG (peak 5); cis-crocins (peaks 7, 8). Picrocrocin (peak *).

$\lambda=250 \mathrm{~nm}$, in which the less intensity of the corcin peaks is recognizable. Moreover, Figure 6 clearly shows that no picrocrocin peak is detected in cheese samples, probably due to its degradation during cheese making.

According to the literature, this is one of the few times in which saffron functional compounds are identified and quantified in dairy products. The first attempt was made by Licón et al. ${ }^{14}$ who studied saffron color in ewe milk at different fat levels and saffron concentrations. They demonstrated that color changes were statistically significant when increasing the fat content of milk, as well as saffron concentration. Later Licón et al. ${ }^{13}$ made pasteurized pressed ewe milk cheeses with saffron in order to study compositional, microbiological, textural, color and sensory characteristics of cheeses in relation to saffron concentration and ripening time. The main variable of these works ${ }^{13,14}$ was color, which contribution could not only be ascribed to saffron, but also to different compounds of dairy products and their interactions with saffron, such as, for example, the fat content of milk.

\section{Conclusions}

This application was employed to determine crocins both in saffron powder and in the different analyzed cheeses made with this spice, because food items containing saffron are expected to provide an added value for consumers.

The UHPLC method described above represents a less time and solvent consuming methodology to simultaneously determine, and with a short time of analysis (16 min), low amounts of crocins and picrocrocin. This method also provided further information about saffron quality used during cheese making, as it is able to discriminate between trans and cis-crocins, important for saffron therapeutic properties.

Chromatographic profiles of different cheeses made with saffron reflected the chromatographic profile of saffron, differing only in quantitative composition. Since no picrocrocin was detected in the analyzed cheeses, further studies are needed to determine picrocrocin and safranal in cheese, in addition to crocins, by using UHPLC.

The presence of some of these molecules in all analyzed samples encourages the use of this spice in the dairy supply chain, due to its potential protective action on human health. Unfortunately, no information are most often available on the saffron employed, because of a traditional and/or niche production of cheeses made with this spice, therefore it would be worth knowing the exact amount of saffron added in order to evaluate the real amount of saffron functional compounds in these food items. Further studies are also needed to evaluate the potential healthy properties of the remaining saffron compounds in cheese.

\section{Acknowledgments}

This work was financially supported by the Italian Ministry "Ministero delle Politiche Agricole, Alimentari e Forestali (MiPAAF)" within the Project: TERRAVITA (D.M. 25870/7303/2011). The authors thank Dr Corrado Costa (Consiglio per la ricerca in agricoltura e l'analisi dell'economia agraria, Centro di ricerca Ingegneria e Trasformazioni Agroalimentari (CREA-IT)) for his statistical support. 


\section{References}

1. Akowuah, G. A.; Htar, T. T.; J. Nat. Prod. 2014, 7, 05.

2. Alavizadeh, S. H.; Hosseinzadeh, H.; Food Chem. Toxicol. 2014, 64,65 .

3. Alonso, G. L.; Salinas, M. R.; Garijo, J.; Sánchez-Fernández, M. A.; J. Food Qual. 2001, 24, 219.

4. Iborra, J. L.; Castellar, M. R.; Canovas, M.; Manjón, A.; J. Food Sci. 1992, 57, 714.

5. Sànchez, A. M.; Carmona, M.; Zalacain, A.; Carot, J. M.; Jabaloyes, J. M.; Alonso, G. L.; J. Agric. Food Chem. 2008, $56,3167$.

6. ISO 3632-2:2010: Spices - Saffron (Crocus sativus L.) - Part 2: Test methods, International Organization for Standardization, Geneva, Switzerland, 2010.

7. Alonso, G. L.; Salinas, M. R.; Esteban-Infantes, F. J.; SánchezFernández, A. M.; J. Agric. Food Chem. 1996, 44, 185.

8. Carmona, M.; Zalacain, A.; Sánchez, A. M.; Novella, J. L.; Alonso, G. L.; J. Agric. Food Chem. 2006, 54, 973.

9. Tarantilis, P. A.; Tsoupras, G.; Polissiou, M.; J. Chromatogr. A 1995, 699, 107.

10. Zalacain, A.; Ordoudi, S. A.; Blazquez, I.; Diaz-Plaza, E. M.; Carmona, M.; Blazquez, M. Z. I.; Tsimidou, M. Z.; Alonso, G. L.; J. Agric. Food Chem. 2005, 53, 9337.

11. Zougagh, M.; Simonet, B. M.; Rios, A.; Valcarcel, M.; J. Chromatogr. A 2005, 1085, 293.

12. Assimiadis, M. K.; Tarantilis, P. A.; Polissiou, M. G.; Appl. Spectrosc. 1998, 52, 519.

13. Licón, C. C.; Carmona, M.; Molina, A.; Berruga, M. I.; J. Dairy Sci. 2012, 95, 4263.

14. Licón, C. C.; Carmona, M.; Rubio, R.; Molina, A.; Berruga, M. I.; Dyes Pigm. 2012, 92, 1355.

15. Librán, C.; Licón, C. C.; Serrano-Diaz, J.; Carmona, M.; Berruga, M. I.; Dairy Sci. Technol. 2014, 94, 83.

16. Licón, C. C.; Carmona, M.; Berruga, M. I.; Int. J. Dairy Technol. 2015, 68, 399.

17. Hosseinzadeh, H.; Abootorabi, A.; Sadeghnia, H. R.; DNA Cell Biol. 2008, 27, 657.

18. Papandreou, M. A.; Kanakis, C. D.; Polissiou, M. G.; Efthimiopoulos, S.; Cordopatis, P.; Margarity, M.; Lamari, F. N.; J. Agric. Food Chem. 2006, 54, 8762.
19. ISO 707|IDF 50: Milk and Milk Products - Guidance on Sampling, International Organization for Standardization \& International Dairy Federation, Geneva, Switzerland, 2008.

20. Eurachem Guide; The Fitness for Purpose of Analytical Methods: A Laboratory Guide to Method Validation and Related Topics, 2nd ed.; LGC (Teddington) Ltd.: Teddington, UK, 2014. Available at https://www.eurachem.org/index.php/publications/ guides/mv, accessed in March 2017.

21. Hammer, Ø.; Harper, D. A. T.; Ryan, P. D.; Palaeontol. Electron. 2001, 4, 9; http://folk.uio.no/ohammer/past/, accessed in March 2017.

22. Lozano, P.; Castellar, M. R.; Simancas, M. J.; Iborra, J. L.; J. Chromatogr. A 1999, 830, 477.

23. Caballero-Ortega, H.; Pereda-Miranda, R.; Abdullaev, F. I.; Food Chem. 2007, 100, 1126.

24. Kyriakoudi, A.; Chrysanthou, A.; Mantzouridou, F.; Tzimidou, M. Z.; Anal. Chim. Acta 2012, 755, 77.

25. Sarfarazi, M.; Jafari, S. M.; Rajabzadeh, G.; Food Anal. Methods 2015, 8, 2273.

26. García-Rodríguez, M. V.; Serrano-Díaz, J.; Tarantilis, P. A.; López-Córcoles, H.; Carmona, M.; Alonso, G. L.; J. Agric. Food Chem. 2014, 62, 8068.

27. Lage, M.; Cantrell, C. L.; Sci. Hortic. 2009, 121, 366.

28. Masi, M.; Taiti, C.; Heimler, D.; Vignolini, P.; Romani, A.; Mancuso, S.; Food Chem. 2016, 192, 75.

29. Wang, Y.; Han, T.; Zhang, X. G.; Zheng, C. J.; Rahman, K.; Qin, L. P.; Chromatographia 2009, 70, 143.

30. Orfanou, O.; Tsimidou, M.; Food Chem. 1996, 57, 463.

31. Chemat, F.; Huma, Z.; Khan, M. K.; Ultrason. Sonochem. 2011, 18,813 .

32. Indyk, H. E.; Edwards, M. J.; Woolard, D. C.; Food Chem. 1996, $57,575$.

33. Kromidas, S.; HPLC Made to Measure: A Practical Handbook for Optimization; Wiley-VCH Verlag $\mathrm{GmbH} \& \mathrm{Co}$. KGaA: Weinheim, 2006.

34. Vickackaite, V.; Romani, A.; Pannacci, D.; Favaro, G.; Int. J. Photoenergy 2004, 6, 175.

Submitted: March 30, 2017

Published online: July 13, 2017 